\title{
La mise en valeur de la vallée du Sào Francisco
}

\section{Land development in the Sào Francisco valley}

\author{
P.AR P. PELTIER DE QTTEROZ WT S. SEREIBRENIO'K
}

\begin{abstract}
Objectifs et aspril du pojet, ses particntarites. Plan et proframmes des réalisations.

Etudes et releves aériens, pédologiques, géologiques, hydrographiques; itudes économiques et sociales.

Problemes relatifs an flenve et aux régions enmronnantes : riqularisation, navigation, equipement hydro-electrique; aménagements hydroagricoles; communications, assainissement; faclears humains; équipement sanitaire; reboisemenl.
\end{abstract}

\begin{abstract}
Purpose and design of the project, ils portirniar fentures. Plan and programmes for atewelopment.

Aerial reconnaissance and survelys, pedology, geology, hydrograply, economic and socist studies.

Problems concerning the riber and surrounding regions: regularisation, nabigation, hydro-electric equipment, hydro-agrioullaral developments, communication, drainatge, human factors, sanitary equipment, afforestation.
\end{abstract}

\section{I. - Le São Francisco sous l'Empire et pendant la République}

Le bassin du Săo Franciseo retient depuis longtemps l'attention des pouvoirs publics en raison de sa position géographique et des tris larges possibilités icononiquues qu'il présente. L'empereur Don Pedro II, dès 1852, organisait des campagnes d'exploration et des commissions d'ctudes. Apres une période d'efforts sporadiques, le Président Vargas donna, à partir de 1930, une nouvelle impulsion aux travaux d'exploration et à la mise sur pied de grands projets. Cette phase préliminaire, marquéc notamment par l'ćlaboration d'un progranme d'amélioration de la navigation et par la constitution de la Compagnie Hydroćlectrique du São Francisco (concessionnaire du troncon compris entre Juazeiro et Piranhas), s'achève le 15 décembre 1948 avee la création de la a Commission de la vallée du Sío Francisco », chargée de traduire dans les faits l'article 29 de l' "Ato das disposiçoes constitucionais transitorias $\gg$ selon lequel * le gouvernement fédéral s'engageait it tracer et à exéculer dans un délai de vingt ans. a compter de la date de promulgation de celte disposition, un plan de mise en valeur complete des possibilités économiques du fleuve Sĩo Francisco et de ses affuents; plan dans lequel il investira annuellement une somme au moins égale à $1 \%$ des revenus publics $\gg$.

Alors que se dessinail le cadre devant envelopper l'ensemble des études et des travaux concernant ce gigantesque chantier, te programme en cours d'exécution, loin d'être ralenti, élait lui-mème amplifié dans la mesure où il préparait et rendait possible la tâche de la notvelle Commission.

\section{II. - Le caractère national du projet et ses objectifs}

Créce à la fin de 1948, la Commission de la vallée du São Francisco s'est occupée, en premier lieu, d'élaborer le plan général de dévelop- 
pement économique de la région, conformémenl au premier aspect des dispositions constitutionnelles. Dans ce travail, la direction de la Commission a toujours bénéficié de la collaboration spontanée de tous les services publics et des aulres organismes intéressés au développement de la région.

Considérant les puissantes raisons politiques qui avaient amené les représentants de la Constitution de 1946 à inclure l'aménagement économique du São Francisco dans les problèmes de aractere national, la Commission, pour mener a bien sa tâche initiale, s'ellorça de réunir une equipe de techniciens capables d'étudier, de disruter, et de peser, avec une liberté absolue, la solution des problumes de cette région. Le nombre considérable d'éludes scientifiques, techniques et culturelles, réunies dans les mémoires et annexes du projet, que détient le Congrès national, montrent d'une façon éclatante que les solulions proposies pour les différents problèmes de la région onl eu pour base à la fois une large connaissance des besoins locaux acquise directement sur place et le déponillement quasi complet de la bibliographie concernant le bassin du São Francisco. Ceci permettait à la Commission d'chablir, sous forme de projets d'ouvrages et de programmes de travaux, la structure d'un plan d'aménagement général, première étape vers le projet que la région exigera plus tard.

Dans l'exécution de cette première tâche, Ia Commission de la vallée du São Francisco fut suidée par trois considerations fondamentales, trois axes de reférence dont elle ne pouvait s'icarter, à savoir :

-... L'espril dans lequel les représentants de la Constitution décidèrent la mise en valeur du hassin du São Francisco;

-- Les conditions imposées par le texte de l'article 29 de l'Acte des « Disposições Constitucionais Transitorias $»$;

-... Et les particularités de la technique moderne d'ćtude des bassins hydrographiques;

Ce sont ces facteurs que nous allons analyser en détail.

\section{L'ESPRIT DU PROJET}

La raison qui amena les représentants de la Constitution de 1946 à choisir le bassin du Săo Francisco pour le faire bénéficier d'un traitement spécial, dans le cadre de notre structure territoriale, et lui permettre un rapide développement, n'était pas seulement l'ambition de mettre en valeur une vaste région du pays.

Sans tenir compte de sa «position», facteur géographique qui donne au São Francisco une part essenticlle de son importance, il est hors de doute que ce fleuve, qui développe la majeure partie de son cours parallelement au littoral, n'avait pas la vocation d'un fleuve de pénétration vers l'intérieur.

C.essant d'être navigable très près de son embouchure, coupé par une série de rapides et de chutes, environné de vastes territoires semi-arides, ses rives ne farorisent guère une occupation humaine continue.

Tout au long de la vallée du São Francisco, la concentration démographique n'atteint un degré raisonnable que dans sa partie inférieure, où le fleuve prend un caractère de "fleuve de pénétration », et dans sa partic supérieure, non plus à cause de l'influence directe de ses eaux, mais grâce à la valeur des ressources minérales el à une agriculture pionnière exploitées par des hommes émigrés d'autres régions et d'autres bassins.

Sur le moyen São Francisco, sur plus d'un millier de kilomètres, les rives du grand fleuve, pourtant propices au défrichement et à l'immigration, sont demeurces quasi inhabitées, faute de cet indispensable pouvoir de fixation humaine que les grandes vallées possèdent généralement dans les autres continents. Malheureusement, cetle particularite se manifeste justement dans le tronçon du grand fleuve qui relie le nord au sud du pays.

La pénétration sur loute l'étendue du littoral brésilien parallele au bassin du moyen São Francisco n'ayant pas été faite, l'occupation humaine est faible et n'a pas eu le pouvoir d'expansion qui lui eût permis d'élargir une zone homogène de population jusqu'à l'extrémité du fleuve.

La nécessité d'intensifier les échanges entre le nord et le sud du pays par l'amélioration de la navigation intérieure, la nécessité de peupler les rives du grand fleuve d'une manière plus dense et de les rendre économiquement stables, la nćcessité enfin d'ćlever le niveau de vie des populations et d'implanter une civilisation riveraine qui soit une synthèse, une transition, une liaison entre les civilisations qui se développent au nord-est, au centre et au sud du pays, telles furent les raisons qui amenìrent les représentants de la Constitution de 1946 a envisager le problème de la mise en valeur du São Francisco comme étant du plus haut intérêt politique pour la nation : en effet, l'occupation effective du grand fleuve, el son développement économique, supprimeraient le vide qui sépare encore actuellement ces civilisations. Ceci donne donc un aspect objectif à l'effort qu'il faut encore fournir pour affermir l'Unité Nationale, car, lorsque le São Francisco se sera transformé en une contrée de population fixe, en une région dévelop- 


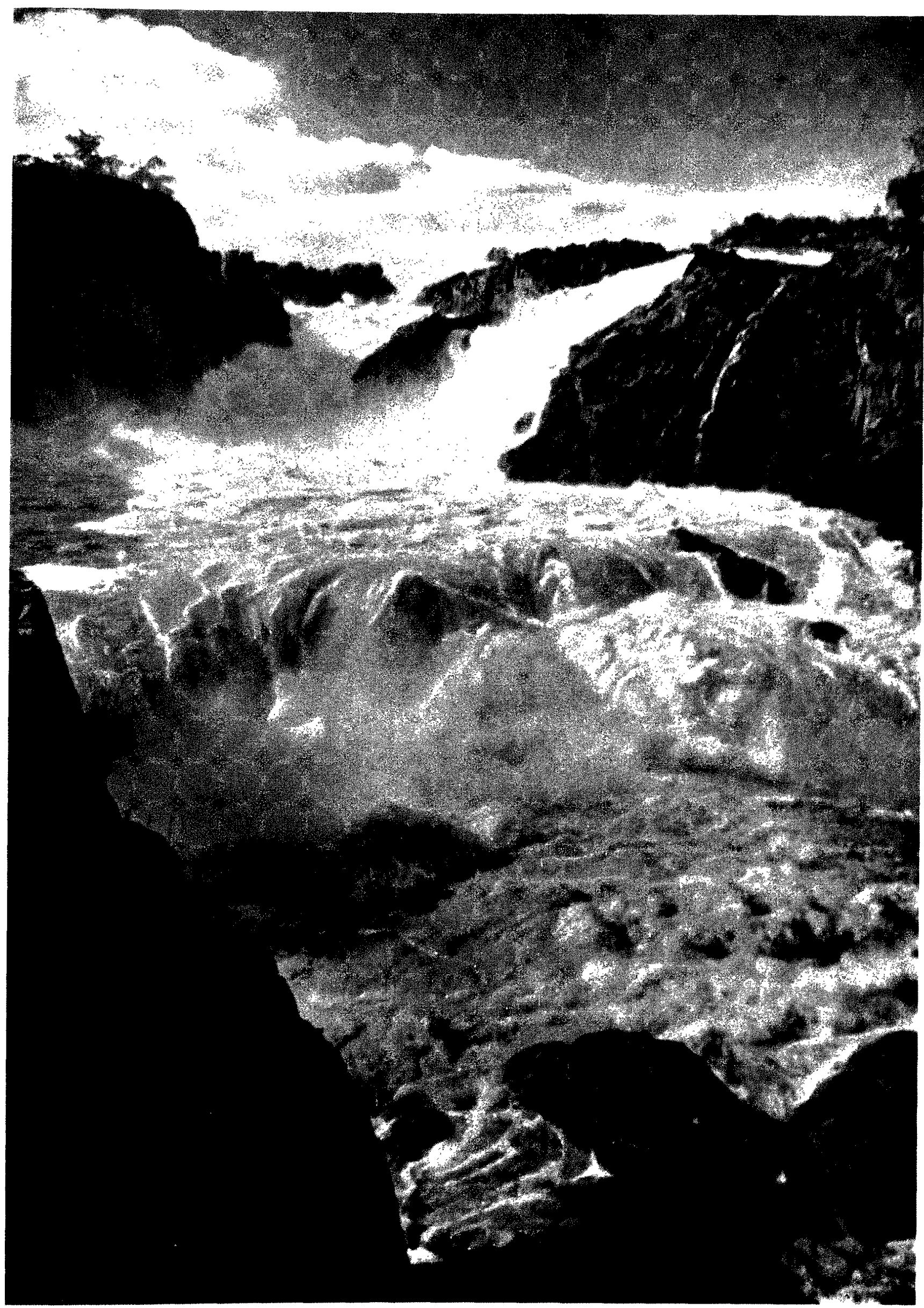

Fig. 1. - Chutes de Paulo Afonso sur le São Francisco. 
pée el en progries, quand il sera à mème d'altirer cette population an lieu de la roir émigrer, une hase matérielle solide sera donnce à l'tnion bresilienne.

La mise en valeur de la vallée du São Franciseo a done pour objet primordial, dans le cadre de lat politique nationale, d'affermir l'unite du pays, laxe du grand fleuve, par sa position ségriphique, servant de trat d'union entre les populations du Brésil. C'est la raison pour laquelle le São Francisco doit devenir réellement le facteur de l'unité nationale, ce qui se produira dans un proche avenir, grâce à l'avancement continu des travaux qui se trouvent actucllement en cours d'exécution.

\section{LES ORJECTIFS DU PLAN}

Calqué sur le texte de l'article 29 de l'Acte des «Disposicoes Constitucionais Transitórias», l'article 7 de la loi $n^{\circ} 541$ du 15 décembre 1948 définit, dans une première partie du projet, les objectifs du plan sénéral d'aménagement; la Commission de la vallée du São Francisco, d'apris celle disposition légale, doit déterminer exactement ces objectifs sous forme de projets et de programmes specifiques.

Le règlement de la Commission, approuvé par le décrel $n^{\circ} 26476$ du 17 mars 1949 , modifié par le décret n" 27301 du 10 octobre 1949 , et remanié par le décret n" 29807 du 25 juillet 1951 , comprend les objectifs suivants : la régularisation du régime fluvial; le contrôle et l'utilisation des eaux; l'amélioration des conditions de navigabilité du fleuve São Francisco, de sa barre el de ses affluents; la mise en valeur de son potenticl hydro-électrique: le développement de l'irrigation et des ouvrages de retenue; l'aménagement des porls fluviaux; l'extension du sysicme régional de lransport; l'amélioration "du trafic fluvial; le développement du réseau de communications: l'assainissement des centres urbains et des zones rumales; la protection des rives el des localités riveraines contre les inondations ef l'érosion; le dégagement des cours d'eau et le drainage des marais et des étangs: les travaux d'urbanisme régional; l'exploitation a la protection des richesses minières, de la faune el de la flore: le reboisement et l'aménagement rationnel des terres; le développement de la production; l'accroissement de l'immigration et de la colonisation; l'instruction et l'enseignement professionnel; la protection sanitaire et l'aide à la population; la défense des intérêts collectifs, au besoin par l'expropriation des étendues nécessaires.

\section{I. - Particularités du projet}

\section{SoN tiNitr.}

La mise sur pied d'un tel programme, puis son exicution, exigeatent avant tout le souci permanent d'une synthèse vigoureuse préservant les traits généraux $d u$ bassin et évitant la lransformation du projet en un amoncellement de plans locaux éparpillant inconsidérément les eflorts.

Or, l'ensemble des régions intéressées comporte un trait d'union, un pôle d'intérêt commun, un probleme central qui leur imprime un caractère d'unité remarquable: l'eau. Cette communion d'intérêt dans la vie des grandes vallées suggère évidemment l'objectif central, facteur d'unilé, vers lequel peuvent s'orienter les études : la maîtrise des eaux fluviales, leur exploitation rationnelle et l'organisation de leur influence sur la vie des populations riveraines.

\section{S.A COMPLEXITÉ.}

Contrastant avec la simplicité de l'énoncé du principe fondamental régissant l'aménagement des hassins hydrographiques - maîtrise de l'eau et rationalisation de son usage --, des difficultés ćnormes surgissent quand on passe au projet proprement dit.

Il n'y a pas de nommes rigides ni de critères uniformes à suivre, ear les vallées ne sont jamais les reproductions fidiles les unes des autres.

Dans le cadre général du principe suivant lequel de tels plaus d'aménagement doivent être conçus pour développer intégralement, et d'une façon coordonnée, loutes les ressources du bassin au bénéfice de l'ensemble de sa population, il y a place pour un tris large éventail de circonstances antagonistes, et par conséquent de solutions al de rariantes.

Une solution unanimement acceptée pour laménagement des vallées est la solution à «fins multiples », dans laquelle les projets isolés doivent envisager le développement de toutes leurs possibilités immédiates ou futures.

Ainsi, par exemple, on devra prévoir l'utilisalion de chaque réservoir d'accumulation et ses répercussions possibles sur la régularisation du régime fluvial, l'amortissement des crues, l'amélioration de la navigation, la production d'énergie clectrique et l'irrigation des terres riveraines.

Par-delà la complexité qu'engendre l'étude d'un tel ensemble d'objectifs - ils se contrarient souvent et il est difficile d'estimer leur importance respective dans l'étude de la solulion la plus adéquate - il ne faut pas négliger l'influence de chaque ouvage projeté sur les autres, qu'ils existent ou soient encore à l'étude. 


\section{LAS NUANCES.}

On avait encore a lenir comple du fait que la vallée du São Francisco, avec ses $630.000 \mathrm{~km}^{2}$ de superficie, n'est pas, à strictement parler, une région homogine, malgré l'influence unificatrice du fleuve.

Dans son bassin existent des contrastes : dans le relief, la géologie, le climal, la végétation, la répartition des forces hydrauliques, ainsi que dans les activités économiques et les conditions de vie de la population.

On comprend dès lors que si, d'unc part, ce bassin doit être considéré comme une unité géon graphique, comme la base territoriale d'un aménagement d'ensemble, on doit, d'autre part, dans ce projel, faire une étude pour chacune des régions naturelles plus petites qui se caractérisent à l'intérieur du bassin.

On en déduit la mison pour layuelle la Commission, dans le projel général du São Francisco, a non seulement prévu, dans leur ensemble el suivant lordre de priorité nécessaire, les points essentiels caractéristiques de l'aménagement d'un bassin hydrographique - points qui sont un lacleur d'unification de tous les problèmes particuliers -- mais a encore cherché à coordonner les activités spécifiques de chaque rigion, en tenant compte chaque fois des aspects de leur développement íconomique, de leur industrialisation, de leur essor agricole et de l'exploitation de leurs richesses naturelles, mais sans jamais perdre de vue le thème principal du projet, la maîtrise de leau : il est certain, en efret, que, faute de s'être assuré ce facteur d'unité, il serail impossible d'organiser l'occupalion de ce territoire, d'y développer la production et le commerce, et d'élever le niveau de vie de ses habitants.

\section{IV. - Le plan général et les programmes des travaux}

On ne saurait trop insister sur le caractère extrêmement général du plan, dont la mise sur pied a été confiéc à la Commission et qui dépassait de beaucoup le cadre d'un simple plan d'électrification ou d'irrigation. De même, l'importance primordiale, le caractire absolument prioritaire el impératif de la régularisation du régime fluvial ne souffrait guère de discussion. Enfin, sur le plan national, le développement de cette tâche ne peut manquer d'être suivi avec beaucoup d'attention, en raison des lecons qu'elle peut comporter pour l'avenir et pour l'aménagement éventuel d'autres régions du pays.

L'ensemble des programmes d'aménagement lut réparti entre quatre plans quinquennaux : pour des raisons d'ordre financier, le premier de ces plans, dont le montant total s'élevait à 1 milliard 743 millions de cruzeiros, dut ètre par la suile assez sérieusement réduit. C'est ainsi qu'un nouveau premier plan quinquennal, se montant seulement a 1 milliard 80 millions de cruzciros, etait approuve par le Président Vargas le 12 oclobre 1951 el soumis au Congres national le 23 du même mois.

En élaborant ce programme, la Commission tint comple de la nécessité qu'il y avait à accorder la priorité - suivant leur degré d'urgenceaux travaux qui, d'une part, représentaient des améliorations déjà en cours et qui, d'aulre part, avaient pour but lexécution d'ouvrages fondamentaux et indispensables au diveloppement économique de la région.

Le début du promier plan quinquennal ayant élé fixé au 1'r janvier 1951, les travaux déjà en cours constituerent une sorte de prolongement du « plano de emergencia » (plan d'urgence) lancé quelques années plus tòt, el permit à lia Commission de commencer immédiatement la mise en ouvre de son programme.

Ce programme ne saurait être compare a certains aménagements a buts multiples réalisés ailleurs dans le monde, aux U.S.A. en particulier: d'une part, le cadre économique de la région ne serait absolument pas adapté à une telle politique; d'autre part, ce serait manifestement trahir l'esprit dans lequel les autorites fédérales ont conçu cette ouve. L'esprit dans lequel sont menées les études et les travaux rappellerait plutòt celui qui préside à l'aménagement de certains territoires de colonisation, íconomiquement sous-développés.

\section{V. - Etudes générales et Relevés}

En s'organisant, la Commission de la vallée du São Francisco chercha à réunir en premier lieu toutes les éludes, observations, enquêtes et recherches qui avaient été faites dans cette région par de nombreux technicions de nationalités diverses, en complétant ces éléments, quand c'était nécessaire, par de nouvelles études, afin de pouvoir, appuyés sur des données sûres, préparer son plan général d'aménagement.

Cependant, la Commission poursuit progressivement, d'année en année, les études que rendent nécessaires l'élaboration détaillée des projets prévus dans le plan.

Ces études comportent en particulier des relevés aériens, pédologiques, géologiques, hydrographiques, hydrologiques et géographiques du bassin du São Francisco, ainsi que des études de communauté, menées parallèlement à l'exploration économique et sociale de la vallée. 


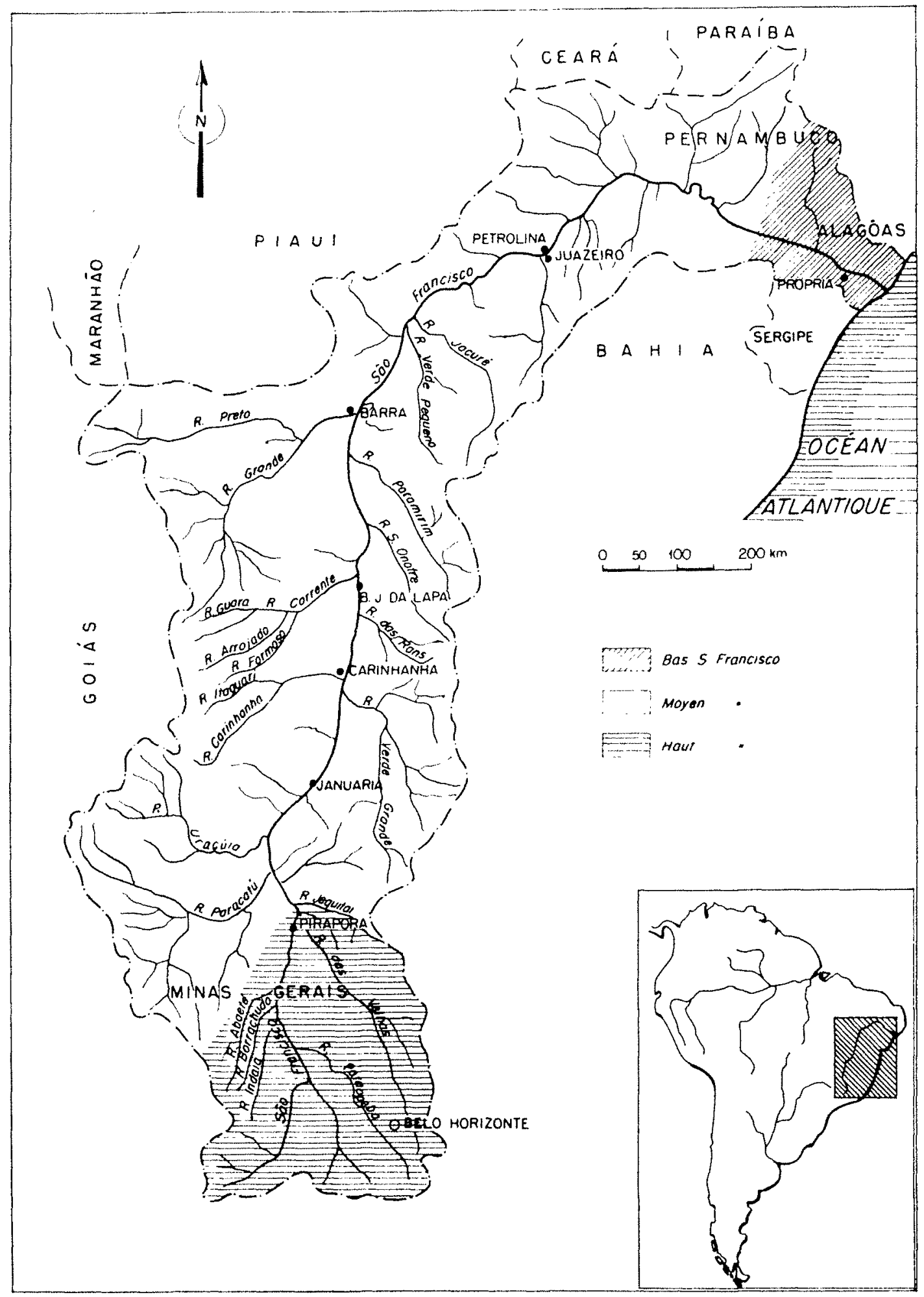

FIG. 2.

Bassin du

Sĩo Franciscu.

\section{Photographie aérimnne.}

Le relevé aérien de la région comprend, d'après le contrat passé dès le début des travaux avec les « Servicos Aerofotogramétricos Cruzciro do Sul S.A. », la photographie aérienne de tout le bassin hydrographique du São Francisco, la restitution aérophotogramétrique, pour l'obtention de cartes topographiques, des sections navigables du fleuve principal et de ses affluents (a l'échelle de $1 / 5.000$ ) et les cartes (à l'échelle de 1/25.000) nécessaires aux projets d'ouvrages 
Fig. 3.

Bassins

hydrographiques

des aftiuents

du

São Francisco.

Carte

des contributions

unitaires.

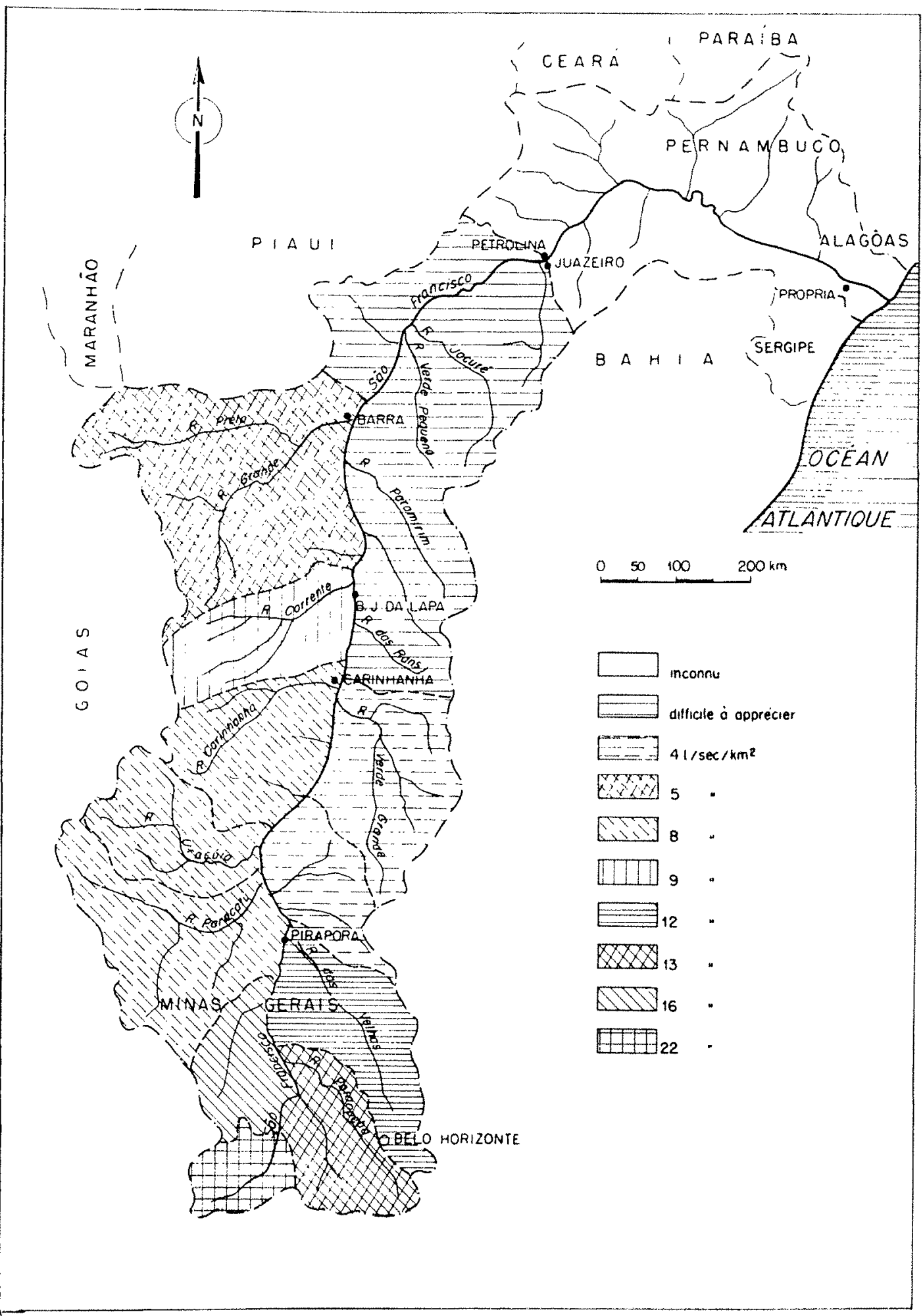

pour toutes les zones intérieures du hassin; enfin, des mosaïques à des échelles appropriées ef des relevés terrestres complémentaires.

Tous les travaux de cartographie sont rapportés aux repères géodésiques, afin que l'on puisse intégrer les relevés obtenus dans la carte topo- graphique du pays, en accord avec le plan de cartographie nationale, et pour tenir compte des consignes $d u$ « Conselho Nacional de Geografia » (Conseil National de Géographie) et du "Serviço Geográfico do Exercito " (Service Géographique de l'Armée); ces diverses dispositions 
permetlent to releve définilif de ces régions el son utilisation dans foules les branches de l'activile nationale.

Le relevé aérien de la vallée du Säo Francisco, destiné a faire connaitre à la Commission, d'une facon détaillée et parfaite, le relief du bassin, représente la plus srosse contribution de ce senre à la carlographic nationale. Jusqu'ici, la photographic aćrienne a couvert $420.000 \mathrm{~km}^{2}$ sur les 630.000 de la superficie du bassin, el on a pu cartographier définitivenent : à l'échelle du $1 / 5.000$, les sections navigables du moyen et du bas São Francisco, avec une surface restituée cle $11.580 \mathrm{~km}=$ à l'échelle du $1 / 25.000$, une itendue de $15.381 \mathrm{~km}^{2}$, à des fins diverses, y compris le calcul de la capacité des bassins de barrages ou d'irrigation.

Parallèlement à ces travaux, durant la période quinquennale 1951-1955, l'entreprise contractanle, en accord avec les programmes préalablement établis, doil également exécuter la restitution aérophotogramétrique de plus de $17.500 \mathrm{~km}^{2}$ a l'échelle du 1/25.000 dans les bassins du São Francisco supérieur, el sur les rivieres: Velhas, Corrente el Grande.

\section{ReLevés pédologiQ̨eEs.}

Desliné essentiellement à renseigner exactement la Commission sur la qualite des terrains dans chaque zone du bassin en vue de son aménagement agricole, le relevé de la carte des sols du bassin du Sĩo Francisco représente aussi une grande contribution a l'ensemble de la pédologie nationale.

Ces travaux, en cours d'exécution par la "Sociedade Civil de Engenharia Quimica e Agricola Llda $»$ à la suite du contrat qu'elle a signé en 1951 avec la Commission, couvrent jusgu'ici une superficie de $480.000 \mathrm{~km}$.

Dans ce but, sur les 23.000 analyses qui sont nécessaires pour recouvir complètement la surface du hassin, pas moins de 16.000 , rialisées suivant le procédé caténaire, ont élé représentées sur 5.333 profils. Le contrat prévoit, en plus des analyses proprement dites, la carlographie de la région.

En plus de ce relevé général de la carte des sols du bassin du Säo Francisco, l'entreprise contractante doit faire d'autres analyses dans des buts precis, principalement dans les régions intéressées par les projets de grande irrigation.

D’après les résultats obtenus jusqu'à présent, nous pouvons affirmer, sans crainte d'erreur, (qu'il existe dans le Säo Francisco des zones de terrains tris fertiles qui, convenablement irriguces, se transformeront en un véritable grenier pour le Brésil car, en agriculture moderne, les régions à irrigation régulière présentent d'énormes avantages.

\section{Frudes ghologloues.}

Le relevé géologique de la région, destine a renseigner parfatement la Commission sur ses réserves minérales, fut commencé en 1949, un accord ayant été passé avec le « Conselho Nacional de Geografia » pour l'exécution des travaux geologiques, entre autres ceux qui se rapportaient au développement écononique et à l'industrie miniere.

Suivant les conclusions auxquelles était arrivé le «Conselho Nacional de Geogralia », il s'avérail nécessaire d'étudier en détail difrérents gisements et de dresser sur-le-champ les avant-projets de quelques exploitations. Ces études portaient principalement sur l'estimation d'un cerlain nombre de gisements : abrasifs naturels ou maticres premicies pour la fabrication d'abrasils synthétiques, chromite, minerais sulfurés pour l'industrie de l'acide sulfurique, minerais de cuivre, de zine, de vanadium ou de plomb, sel semme, sel de polassium, platre, pierres précieuses, calcaire pour l'industrie chimigue on lindustrie du ciment. Les avant-projets portaient sur l'électro-métallurgie, les abrasifs synthétiques, le bichromale de sodium et l'acide sulfurique.

A partir de ces conclusions, la Commission projeta de réaliser ces travaux importants en passant un contrat avec des specialistes, précisant le programme des recherehes indispensibles à l'éclaircissement des différents aspects techniques du problème : existence des matières premières de base pour les différentes industries; procédés industriels les plus appropriés pour la transformation des matieres premières existantes; repartition d'industries on de combinats industriels la mieux adaptée à la région considérée. Ce programme, que le « Departamento Nacional da Producão Mineral » examine en ce momenl, n'a pas encore été approuvé par le Président de la République.

Ces éléments sur les ressources minérales de la vallée du São Francisco iront compléter les renseignements exacts que la Commission doit posséder sur le relief, le sol et les sous-sols de la région, éléments fondamentaux pour l'étude détaillée des phases progressives de l'aménagement économique du bassin.

Dès que la Commission sera en possession de ces données, elle pourra se rendre compte de la viabilité économique de chaque industrie, en pesant les avantages et les inconvénients de leur installation dans la région, d'après le caractère national ou régional de chacune.

\section{RELEÝ́s HYDRographigues.}

Afin de compléter le relevé aérien du lit du fleuve et de préciser, pour la Commission, la 
Fici, 4.

Répartition de la population d'après

lo recensement de 1940 .

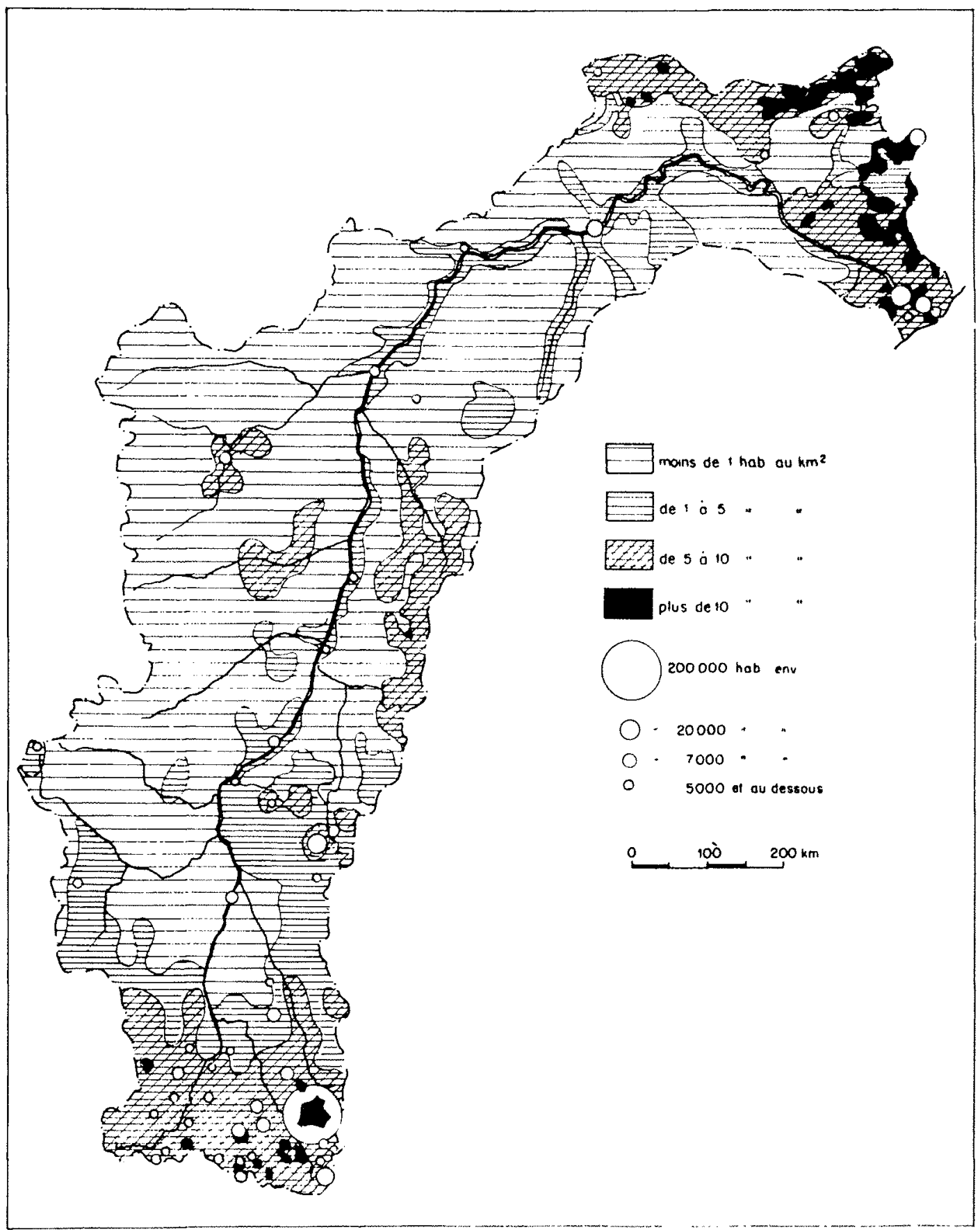

profondeur des diflérents troncons navigables du São Francisco et de ses affuents, une campagne de sondages en rivières ful entreprise aussitôt après la restitution des premières carles aćriennes.

Cette campagne, réalisé suivant la méthode de l'écho, a débuté dans le port de Juazeiro. Poursuivie vers l'amont, elle a atteint un point situé à $283 \mathrm{~km}$ environ, près du port de Pião Arcado, sur le moyen São Francisco.

En dehors de l'exploration du lit du fleuve proprement dit, de nombreux travaux topogra- phiques ou hydrographiques ont pu, d'ores el déjà, être menés à bien sur certains points : rapides de Sobradinho el de Curralinho, aux accés et rives des ports lluviaux, passages difficiles et rapides du noyen et du bas Sĩo Francisco. On a enfin délerminé le profil en long du tronçon de $1.370 \mathrm{~km}$ compris entre les ports de Juazeiro el de Pirapora.

L'ensemble de ces éléments hydrométriques, ou topographiques, est indispensable a la mise sur pied des projets détaillés des ouvrages destines a favoriser la navigation sur ce lleuve et 


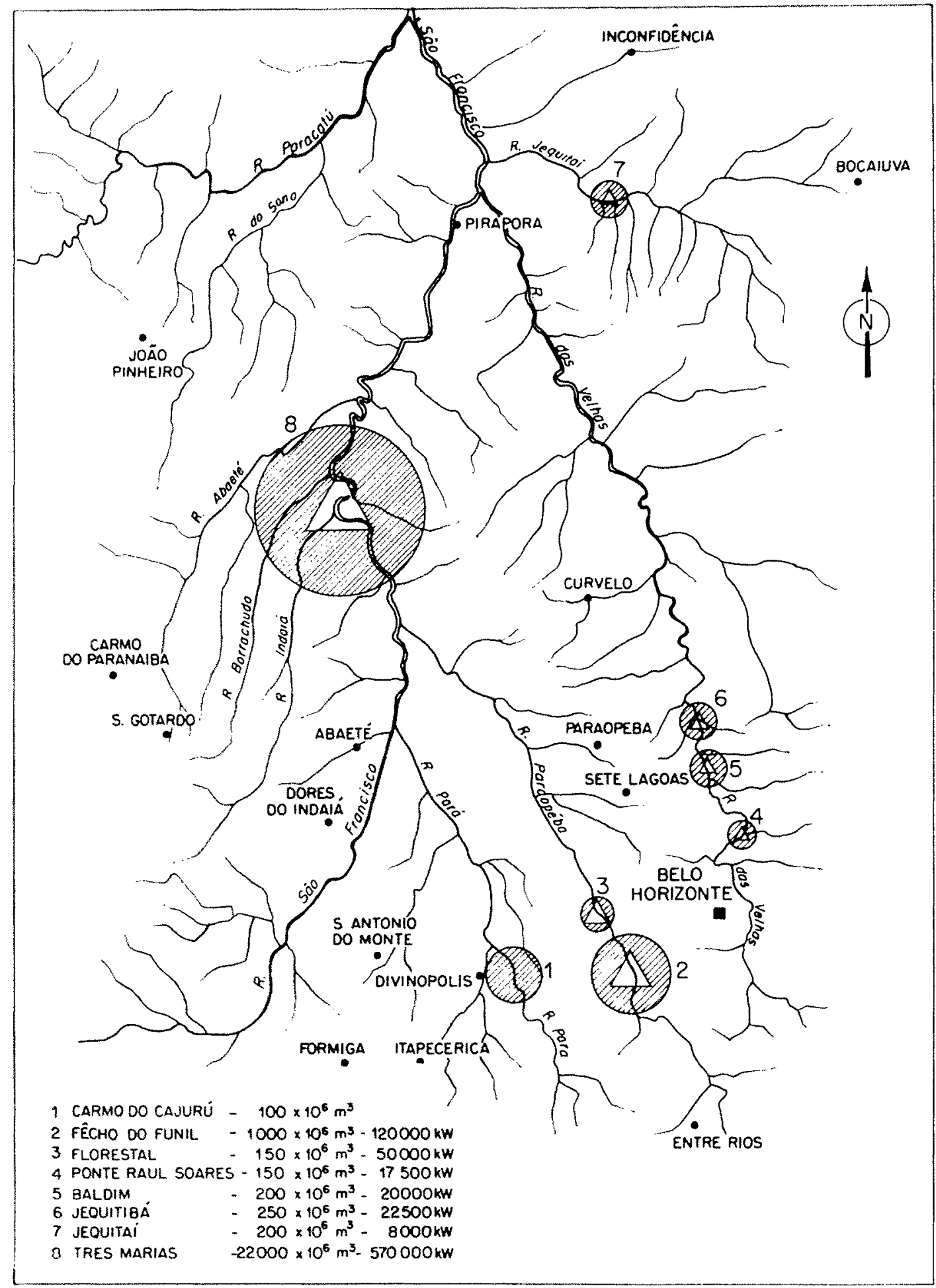

Fic. 5.

Régularisation et equipement du haut

Săo Francisco. a ameliorer les conditions d'accés on d'accoslage des ports fluviaux: ces ouvages devant ètre termines à la fin de la période quinquennale 1951-1955.

\section{Etude hydrologique.}

L'étude hỵdrologique du bassin du São Fran- eiseo, destince à foumir it la Commission les diéments indispensables au projet délaillé des ouvrages de régularisation, est en cours d'exícution par la « Divisio de Aguas do Departamento Nacional da Producão Mineral ». Les travaux en cours tendent à étoffer le réseau des stations hydrométriques existant le long du fleuve, conformément à l'accord passé en 1951 
listi. bi.

Réseau de voies navigables.

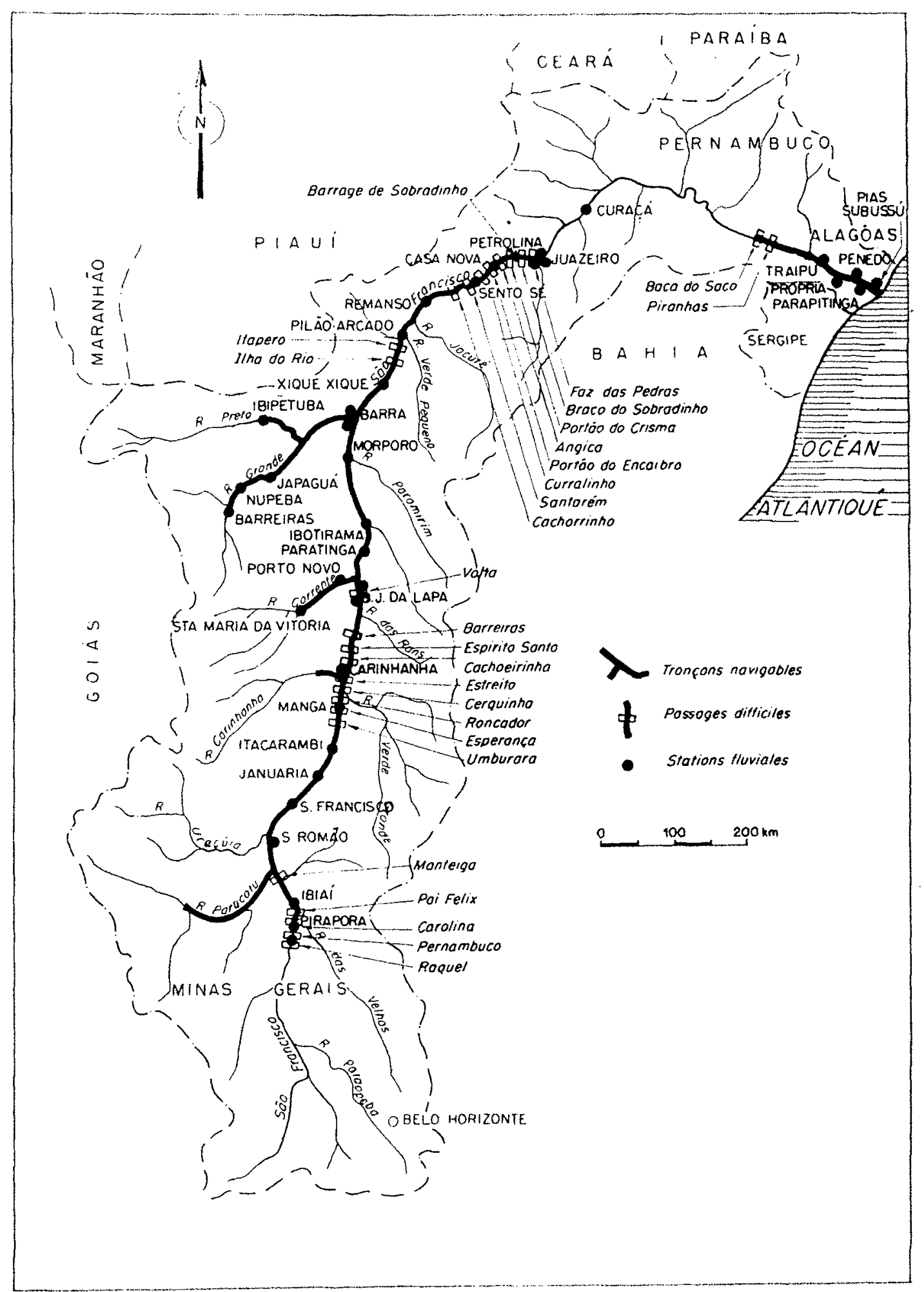

entre de « Ministerio de Agrienlum mission, celle-ci foumissant chaque anméc les fonds nécessaires.

D’un autre côté, la Commission, considérant linsuffisance notoire des données hydrométriques qu'elle possédait sur les bassins secondai- res, signa en 1952 un conlral avee ha "Hirloslosia Comercial Ltola $"$ pour l'installation de 50 nouvelles stations sur les divers affluents et pour la réalisation des premic̀es mesures de débit.

Jusqu'ici, 23 nouvelles stations onl ete instal- 
lées par l'enlreprise contractante ef 283 mesures de débit ont íté faites sur les aftuents. Pendans ce temps, la "Divisão de Aguas» installait 3) stations el réalisail 16 jaugeages daus le fleuve.

\section{ETUdes gEographiques.}

Pour faciliter les travaux relatils à la géographie et à la cartographie du Sảo Francisco, la Commission signa en 1949 un accord avec le «Conselho Nacional de Geografia », qui devait procéder à des études plus poussées de la géographie de la région. Ces études, dont les résultats préliminaires ont été remis en temps utile, ont énormément servi pour l'élaboration du plan général du São Francisco.

Le "Conselho Nacional de Geografia 》 termine actuellement les études géographiques et cartographiques de la région, la remise définitive à la Commission des éléments de cette etude devant se faire à la clôture du hudgot en cours.

\section{ETUdes ECONOMIQLes ET SOCIALES.}

Les eludes dordre social el économique de la vallée du São Francisco furent exécutées sur le budget de 1949 et leurs résultats servirent de base à l'élaboration du plan général. En effet, ces etudes ont trait, d'une façon générale, à la topographie et à l'aspect superficiel des terrains, aux relations entre climat et cultures, à l'élevage, aux voies de communication, aux méthodes agricoles, aux ressources économiques, à l'importation ou a l'exporlation des denrées, aux industries agricoles et manufacturières.

Alin d'approfondir l'etude des groupes humains et à la suite de l'encuête menée, pour le compte de la Commission, sur le budget de 1950 , par le Professeur Donald Pierson, de l' « Institule of Social Anthropology $\gg$ (U.S.A.), le plan 1951-1955 comporte un programme extrêmement détaillé, visant à une meilleure connaissance du milieu, de l'homme et du sol dans la vallee du Sío Francisco.

L'objectif principal de ces recherches, conduites par le professeur Donald Pierson, et dont l'execution fut définie en 1951 par un aceord de la Commission avec l'Ecole de Sociologie et de Politique du São Paulo, est d'obtenir des chantillons representatifs de toutes les divisions du bassin du São Francisco. Un tel objectif rendail nécessaire la connaissance des diflérentes variations relatives à la base écologique, a la société et à la culture.

Le plan général du São Francisco, élaboré par la Commission ef soumis par le Pouvoir exécutif a l'approbation du Congres National, reposait ainsi sur des études, des relevés, des enquêtes, des observations et des prospections qui ont abordé, d'une façon générale, les élémenls principaux du monde physique, économique et social, qui constilue le bassin du São Francisco et qui, d'après ce qui a été expliqué, seronl approfondis dans la mesure où ils s'avereront indispensables à l'exécution rationnelle des projets contenus dans le plan lui-même.

\section{VI. - Le plan: ses problèmes et son exécution}

\section{Thavaux de régulabisation.}

Lanalyse objective des problèmes du São Francisco, et l'étude de l'expérience qu'ont acquise d'autres pays dans l'etude de questions semblables, ont amené la Commission à considérer comme probleme fondamental du projet la régularisation du régime fluvial.

Le bassin du Sio Francisco, conslitué d'un grand nombre de bassins alfuents, grands ou petits, saisonniers ou non, alleint un débit anmuel de 120 milliards de $1^{3}$ et se classe ainsi parmi les grands bassins hydrographiques du monde. Convenablement regularisé el aménagé, il sera un dément sûr de la mise en valeur économique du Brésil et, en particulier, des régions qu'il englobe.

Le projet de régularisation fluviale, devant être exécuté par ćtapes et d'une facon progressive, fut établi en lenanl compte de la nature même de cette entreprise; cependant, il est certain que sa premicre phase comprendra au moins la construction d'un cerlain nombre de digues capables d'éviter les débordements excessifs du fleuve lors des crues.

La Commission, en ce qui concerne le plan de régularisation fluriale, procéda à l'cxamen comsplet du probleme, et elle en lira la conclusion (que le fleuve el ses plus grands afluents permettaient des aménagements rationnels au prix de barrages servant à des fins multiples. Il ne restait plus qu'à définir l'ordre dans lequel ces harrages devaient être construits. En efret, d'apres les donnces concernant l'augmentation du tirant d'eau et le volume des ondes de crue, il serait nécessaire, dans la première phase de régularisation, de retenir un volume d'eau de $\tau$ milliards de $\mathrm{m}^{3}$.

Après une série d'ćtudes spéciales, la Commission décida que la solution du problime de la regularisation fluviale dans le bassin du São Franciseo devail itre orientée de la facon suivanle :

(1) D'abord, construire des réservoirs d'aceumulation dans le hassin supérieur du Sĩo Francisco, en amont de la chute de Pira- 
pora, et dans les bassins des principaux affluents;

b) Ensuite, aprés avoir complétement épuisé les possibilités de relenue dans ces bassins, s'il élait encore nécessaire d'en augmenter le volume, conslruire des barages daccumulation dans le lit mème du Sĭo Francisco, dans son cours moyen.

Tenant compte de ees directives, el considerant que près de $75 \%$ du débit total du bassin du São Francisco provient de la région « minière » de la vallée (Elat de Minas Gerais), la Commission adopta, comme phase initiale de son programme de régularisation : l'aménagement d'une grande retenue sur le cours supérieur du São Francisco, entre les confluents des rivières Indaiá et Abaeté; la réalisation d'un barrage d'accumulation à « Fêcho do Funil », sur la riviere Paraopeba; la constitution d'un réservoir saisonnier à « Carmo do Cajurá », sur le lleuve Para; la croation d'une relenue à Floreslal; ef lat construction d'un ensemble de trois barrages sur la riviere "Velhas ». Celle premiere série de réservoirs doit assurer me retenue supérieure à celle qui avail ele prévue pour la phase préliminaire de régularisation.

Les ouvrages de regularisation qui doivent elre construits dans le haut São Francisco permettront au fleuve, dans son cours moyen el inférieur, de ne pas déborder de son lit normal pendant les périodes de crue, ce qui contribuera a résoudre les problemes suivants:

a) Récupération de la grande bande de terre arable, libéréc aux basses eaux, qui s'étend sur des kilomètres, de chaque còté du fleuve, sur une grande partie de ses affluents;

b) Protection des localités contre les inondations périodiques, ce qui facilikera l'établissencnt de ports fluviaux;

c) Stabilisation du lit, permetlant d'annuler presque complielement les variations de profondeur et favorisant ainsi la navigation;

d) Augmentation du tirant d'eau du chenal it l'éliage, favorisant la navigation et évitant l'exécution d'un grand nombre d'ouvrages fixes disposés dans le lit même du flenve pour améliorer la navigabilité dans les passages difficiles ou les troncons les moins profonds;

(2) Elablissement, d'une manière definitive, des systènes d'électrification, irrigation, transport, etc., indispensables à l'aménagement économique de la région.

Actuellement, dans le cadre de ses travau: pour la période quinquennile 1951-1955, la Commission se préoccupe, en ee qui concerne la régularisation fluviale :

a) D'élaborer le projet définitif du grand barrage sur le cours supérieur du Sío Francisco et de réviser le projel intial du barrage « Fècho do Funil »;

b) D'étudier l'ensemble de la régularisation de la riviere " Yelhas $»$ a lande des trois barrages dejà mentionnés;

c) De construire le barrage d'aceumulation de Cajurtu, qui est en voie d’achèvement.

Comme le but à alleindre est te développement économique de la région, la Commission donne la priorité, dans le projet d'ensemble, à la construction du grand barrage, sur le cours supérieur du São Francisco, dont le plan définiLif a été confié à la "Servix Engenharia Llua».

Cet immense réservoir, qui doit être construit all cour de l'Llat de Minas Gerais, enlye les confluents de l'Indatia el de l'Abacti, où se tromice la cataracte de Tres Marias, comslituera un des plus grands hassins d'accumulation d'Amerique du Sud. Pour une hatuleur de $50 \mathrm{~m}$, il aura une relenue lolale de 14,6 milliards de mi", environ dix fois le rolume des eatux de la Baia de Guanabara, el fournira une puissance de $756.000 \mathrm{ch}$. Le Congres National a déjà prévu, pour le financement de son exécution, pendant la validité du dispositif constitutionnel du Sĩo Francisco, la somme de 100.000 .000 de cruzeiros par an, en plus de son budiget propre.

A lui seul, ce grand réservoir -... la photographie aćrienne de la contrée a jermis de définir l'emplacement du barrage - fournira, pour la régularisation du régime, un volume louble du minimum jugé indispensable pour que cel ouvrage soit compris dans la premiere élape du plan. Il permetlra de transformer le Saro Francisco en un lleuve franchenchl navigable a l'étiage et contribuera a augmenter de $50 \%$ le débil d'éliage du lleuve a Paulo Afonso, qui se trouve a pres de $2.000 \mathrm{~km}$ à l'aval. Il résoudia en ontre definilivement le probleme de lélectrification du haul São Francisco el celui de l'irrigation a grande échelle de la superficie qu’il desservira.

Les études définilives de la régularisation de la rivièe "Velhas » onf fail l'objet d'un contrat, en 1953, entre la Commission et le bureau technique de l'ingénieur (Osear Machad, da Costa; et l'accord relatil a la conslruction du réscrvoir d'accumulation du Cujurì a che passé en 1952 avec le gouvernement de l'latal de Minas Gerais. Ce reservoir doit avoir une retenue d'environ 500 millions de $m^{3}$ et permeltre la pleine utilisation, à l'cliage, des machines déjà installées a la centrale électrique de 
Gafanhoto, dont la puissance passera ainsi de 8.000 à $18.000 \mathrm{ch}$.

Ceci conlirme done que la Commission n'a pas cessé de s'allaquer, depuis le délut, à la solution du problime fondamental du projet, en dudiant la régularisalion du régime fluvial d'une maniere objeclive, respectanl ainsi l'imperatif fondamental de l'élude des hassins hydrographiques.

\section{NAVICATION GeT TRAFIC FUUMAL.}

En ce qui concerne la navigation et le Irafic: lluvial, la Commission s'est orientée vers les objectifs suivants :

a) Amćlioration des conditions de navigabilité du fleuve, de sa barre et de ses affluents;

b) Amplification, modernisation et normalisafion du trafic fluvial.

Au cours de la periode quinquennale de 1951 1955, le programme privoit, entre autres, en ce qui concerne l’amélioration de la navigabilité : l'étude de la riviere Velhas; lexécution des projets dimblioration des passages difficiles et des rapides: lélude de la partie inférieure du moven São Francisco, à l'aval de Juazeiro, et de sa barre: la construction du barrage à écluse de "Braco do Sobradinho ", qui est en roie d'achirement et qui permettra de réduire de $90 \%$ les accidents de la navigation sur le moven São Francisco. accidents qui se produisent précisément sur ce dernier troncon, où le fleuve, sur $9 \mathrm{~km}$ de Iongueur. a une pente de $60 \mathrm{~cm}$ par kilometre au lien de $\pi$.

Dans la partio du programme relative au frafic Iluvial, la Commission estima qu'il était d'une importance fondamentale d'exéculer en premier lien des travaux pour l'augmentation de la capacite de transport. de chargement, de dechargenent et de stockage, avant de réorganiser la flotte fluviale ef les installations a terre correspondantes.

IC plan général du Sĩo Franciseo conclut en montrant la néessile dorganiser une socicté à conomit mixte. pour remplacer les entreprises qui fonctionnent actuellement sur le São Francisco, ce qui éviterait la dispersion des forces at permellmait le réciuipement et la modernisation de la flotte fluviale of des ateliers. ainsi que ha rationalisation des services. mesures indispensables à leffeacite des lmansports et à l'équilibre des operations commerciales. Dans ce hut. le President Gelulio Vargass a public. le 27 anoul 1953. le deeret $n$ " $33.68 \%$ consideranl dutilite publique les mesures dexpropriation contre les entreprises qui travaillent sur le São Francisco.

La Commission a ansi slaboré un programme complet de renranisation du trafic fluvia!, obéissant à des caractéristiques prétablies, et prévoyant l'acquisition de nomvelles unités ef le renouvellement de celles qui existent, l'élablissement de lignes de navigation, la construction de nouvelles installations ef du nouveau chantier fluvial déjà construit à l'île de Fogo.

Quant à l'augmentation de la capacité de chargement, de déchargement et de stockage, dans les ports du moyen et du has São Francisco, la Commission met à exécution, pendant la période quinquennale actuelle, le projet initialement approuvé, qui comporte des ouvrages de protection et d'accostage ainsi que des entrepôts et des services complémentaires.

\section{EQuipement hydro-Électrigue.}

En ce qui concerne la première phase de l'aménagement des sources d'énergie de la région, la Commission a orienté la solution de ce probleme fondamental de la facon suivante:

a) Elargissement de la concession accordee a la Compaginie Hydro-électrique du São Francisco, qui comprendra maintenant toute la partie aval du fleuve principal à partir des rapides de Sobradinho;

b) Organisation d'une nouvelle société à économie mixte, sur le modile de celle qui a été mentionnée plus haut, destinée à la mise en valeur progressive de l'énergie hydraulique du bassin du haut São Francisco;

c) Construction, sous la conduite directe de la Commission, de centrales électriques of de lignes de transport dans la résion sous-développée du moyen São Francisco, lorsque ces travaux seront jugés utiles au développement des moyens de production de la contrée.

Liélargissement de la concession octroyée i la Compagnie Hydro-électrique du Säo Francisco sera accordé, dans les limites des formalités imposees par la legislation en vigueur, lors de l'approbation par le Congrès National du plan général d'aménagement du São Francisco. L'organisation de la nouvelle société à économic mixte pour l'exploitation des ressources du haut São Francisco est actuellement en cours, aux lermes de l'accord passé en 1950 entre le gouvernement de l'Elat de Minas Gerais et la Conuission, la procédure se trouvant i son slade délude final aupres des organismes compélents du * Ministério da Fazenda " Ministere: des Finances).

Quant à la construction des centrales électriques du moyen São Francisco et des lignes de transport correspondantes, la Commission a 
étudié et mis à exécution, pour la période quinquennale 1951-1955, les aménagements suivants :

a) Centrales électriques de Pandeiros, Jequitai, Bambui, Abacté el Pirapora, dans l'Elal de Minas Gerais;

b) Réseau dectrique Formoso-Corrente, y compris les lignes de transmission pour les municipalites de Correnlina, Santa Maria de Vitória, Santana dos Brejos, Carinhanha, Lapa et Paratinga, qui se trouvent toutes dans l'Etat de Bahia;

c) Réseau de transport à partir de Paulo Afonso vers les rigions avoisinantes comprises dans le bassin du São Francisco.

La construction des centrales électriques de Pandeiros et de Correntina, ainsi que celle de leurs lignes de transport respeclives, se trouve actuellemenl à un stade avancé, et le contral pour la construction des installations de Bambui, Abacté et Pirapora vient d'être passé. La centrale de Jequitaí, et le réseau de transport liant Paulo Afonso aux régions avoisinantes comprises dans le bassin, dépendront de la mise au point de leurs projets respectifs; la Commission s'occupe actuellement de l'organisation de la société à économie mixte qui aura à sa charge l'étude de ces installations électriques.

\section{AmÉNAGEMENTS mydRo-dGRICOLES.}

Les éludes réalisées pour la mise en valeur de la région ont montré que l'exploitation du sol ne sera possible qu'en irrigant, ce qui permettra de crér les conditions favorables au développement d'une agriculture moins routiniè et, par conséquent, plus productive.

Considérant que l'établissement de systèmes d'irrigation, grande et petite, constituent le point de départ de la colonisation effective de la région et de la fixation des populations dispersćes, la Commission conclut à la nécessité de ces travaux sous la forme suivante:

a) Exécution, sous la conduite directe de la Commission, des grands réseaux d'irrigalion, avec expropriation des étendues nécessaires, redistribution des terres irriguées el organisation de centres où pourront être développées l'immigration et la colonisation;

b) Intensification de la construction de pelits réseaux d’irrigation, grâce à un régime de coopération entre l'tnion et les agriculteurs riverains.

Pour la réalisation des grands systènes d'irrigation qui encourageront la colonisation, la Commission a choisi des étendues favorables dans les grandes vallees de louest du moyen sio Francisco, en particulier celles des riviores Paracalio, Trucuin, Corrente et Grande.

Les travaux de la « Colonia Agropecolaria do Paracalì $\Rightarrow$ sont actuellement en plein développentent et cetle colonie, dime chendue de \$76.000 ha, dispose à jrésent d'un troupeau de près de 12.000 léles. Les travaux dams la « Colónia Agropecuária do Formoso $\Rightarrow$, dams la vallé de Corrente, sont commencés. Le projet délaillé pour l'irrigation d'une étendue de press do 150.000 ha dans la partie supérieure du Rio Grande est très arancé. Ce projet a été confiè à l'expérience de l'ingéniem italien Luiggi Gallioli. auteur de grands projets d'irrigation en Europe, en Afrique et en Argentine, qui a été spécialement invité dans ce but par la Commission.

En ce qui concerne la petite irrigation, la Commission a signe, en 1951, avec le Ministere de l'Agriculture, en vue de sá réalisation le long de la partic inféricure du moven São Francisco, un accord qui confiait à la "Divisão de Aguas do Departamento Nacional de Producão Mine. ral » l'execution des travaux, moyennant financement effectue chaque anne par la Commission d'après le système du crédit lournant: les travaux se poursuivent régulierement.

Quant à l'utilisation des eaux du São Francisco pour l'irrigation, elle a été ćtudié par la Commission, qui propose, pour le budget en cours, dans un programme à part qu'a igalement approuvé lo Président de ta Ripublique, l'établissement d'un projet d'ensemble pour l'irrigation de loule la parlie da São Franciseo comprise dans le « Poligono das Secas » et s'élendant paliquement depuis le eonfluent du Rio Grande jusqu’à Proprià, qui se trouve déjà dans le bas São Francisco.

Cette bande, de plus de $800 \mathrm{~km}$ de longueur. où se trouvent les petits réseaux d'irrigation qu'établit la « Divisão de Aguas do Ministério de Agricultura », est en cours d'etude par la Commission et son aménagement prosressif permetlra plus tard aux populations du nord-est, disséminés par la rigueur des sćcheresses, do trouver, à l'endroit même qui fut leur herceau, les condilions minima essentielles a leur fixalion définilive.

Afin d'effectuer la sélection indispensable des colons, la Commission a prévu la construction d'une hotellerie à Corinto et de deux postes d'accucil, l'un à Pirapora el l'autre à MonleAzul : l'hôlellerie, prête à alre mise en service, a élé confice a l'Instilul Nalional d'Immigralion af de Colonisalion.

\section{Communications.}

En ce qui concerne les systemes régionnux de communications et de transports, la Commis- 


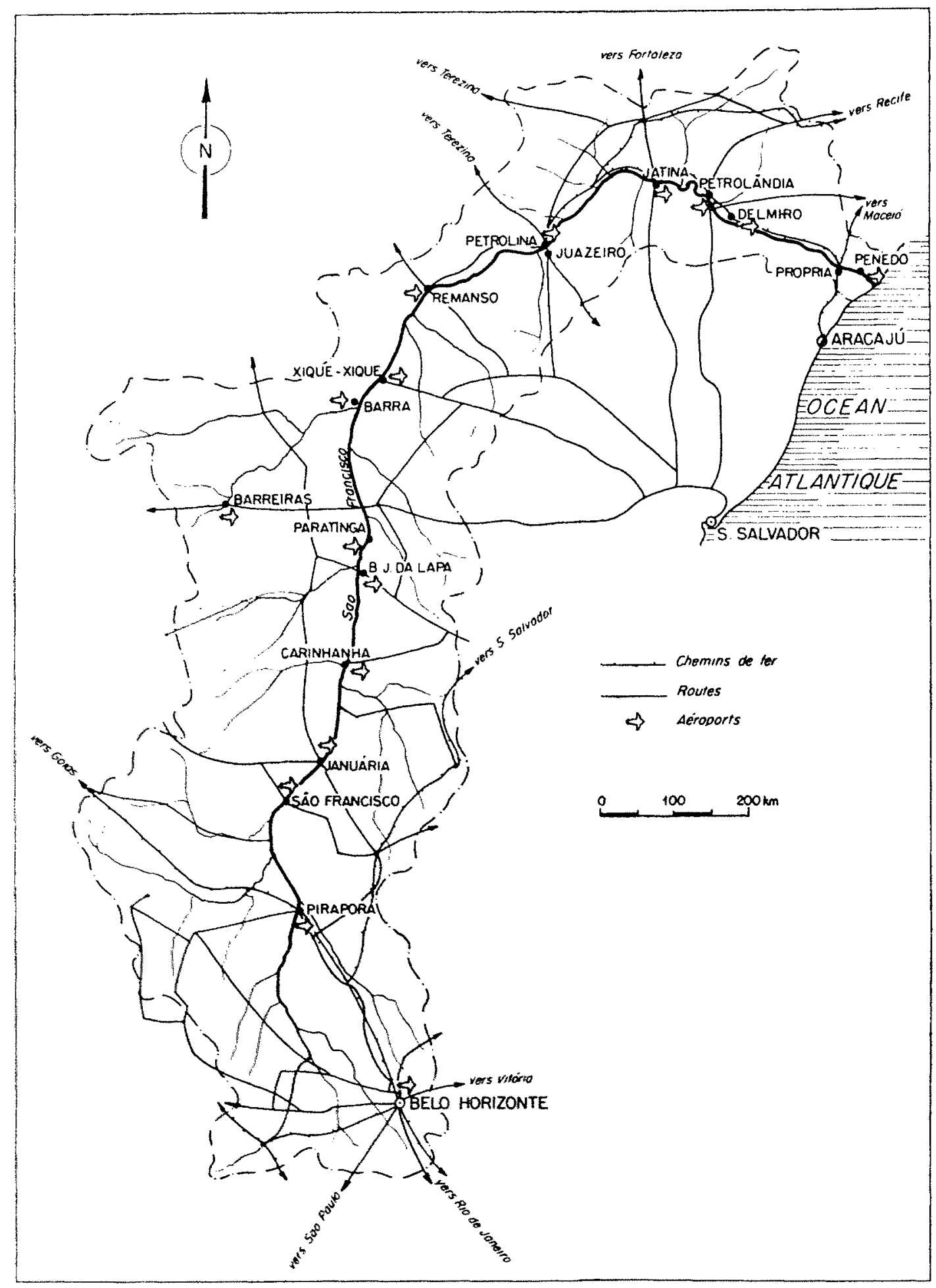

FIti. 7 .

Voics

de communication à l'intérieur. du bassin $d u$ Săo Franciseo.

sion, qui considere comme satisfaisantes les liģnes télegraphiques construites d'apres le \& plano de emergencia du São Francisco, a poursuivi ses travaux avec la construction de routes daces et de liaison. at lexécution douvrages et dinstallations dans les aéroports et les terrains daviation de la e Rota do São Francisco "roule du Sĩo Francisco".
On distingue, parmi les roules les plus imporlantes actuellement en construction, dans l'Elat de Minas Gerais, celles de : Pirapora - Leal Patos; Coração de Jesùs - Brasilia - Sĩo Francisco - Serra das Araras - Sitio de Abadia et Januaria - Montes Claros - Bocaiuva; dans l'Elat de Bahia, celles de : Ihéus - Brumado - Lapa Correntina - Barreiras - Ipirá - Norro do Chapéu 
Fiti. 8

Installations pour

l'augmentation de la

production agricole.

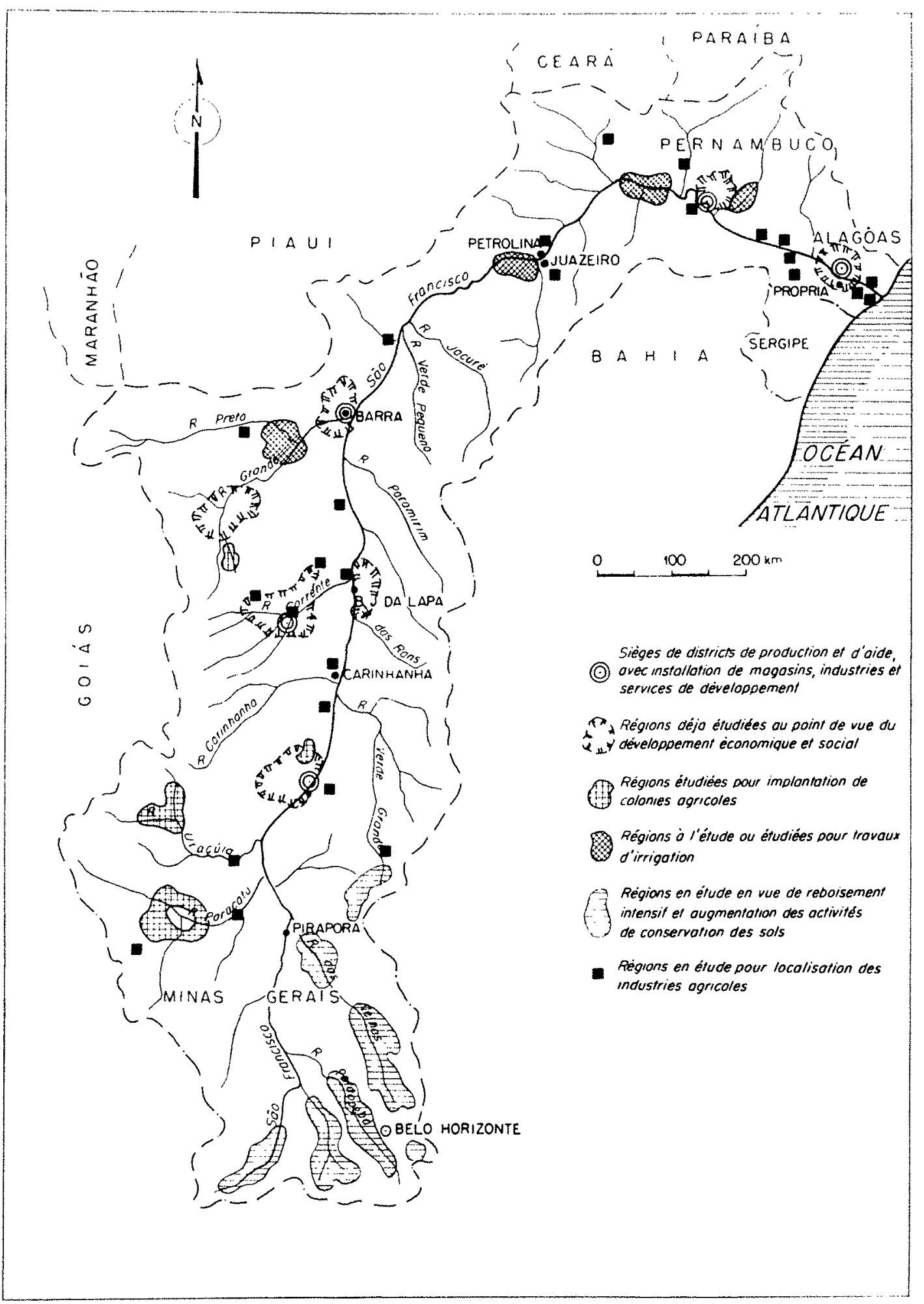

- Xique Xique - Barra - Ibipeluba, Jacobina Remanso - São Raimundo Nonato, et Petrolina Casa Nova - Remanso; dans l'Etat de Pernambuco, celles de : Petrolina - Crato et Petrolândia - Floresta - Jatinã - Cabrobó - Coripós Petrolina; dans l'Etat de Alagoas, celles de :
Santana do lpanema - Pào de Acucar - Piranhas el Arapiraca - Traipù - Pórto Real do Colcyio; enfin, dans l'Etal de Sergipe, celles de : Pôto da Folha - Tamanduà - Gararù el Japoalĩ - Pacaliba - Parapilinga - Néopolis.

La construction de ces routes est a la charge 
des organismes competents des gouvernements des Etats de Minas Gerais, Bahia, Pernambuco, Alagoas et Sergipe; aux termes des accords signés en 1951 et 1952 , ceux-ci recoivent des crédits annuels de la Commission, qui approuve les projets et contrôle les travaux.

D'autre part, la Commission conduit directement l'exécution des ouvrages et des installations effectués dans les aéroports de Pirapora et Januaria, dans l'Elat de Minas Gerais, et, pour les autres aéroports de la « Rota do São Francisco $\gg$, elle travaille en collaboration avec la « $2^{a}$. Zona Aérea do Ministério da Aeronáutica ». Cela permeltra, d’ici peu, de mettre quatorze terrains d'aviation convenablement préparés et installés a la disposition du trafic normal des avions commerciaux sur toute l'étendue de la région comprise dans le bassin du São Francisco.

En ee qui concerne encore les systèmes régionaux de transport, la Commission étudia également, pendant la phase d'élaboration du plan général, les idées et les projets relatifs à l'exécution du réseau ferroviaire national, en essayant de l'harmoniser avec ses propres plans et en suggéranl, pour la partie comprise à l'intérieur du bassin, des agrandissements et des transformations dans les projets existants. En effet, lors des discussions parlementaires au sujet de laménagement du Săo Francisco, à aucun moment on n'avait songé a charger la Commission de la construction des voies ferrées du bassin. La Commission se limitera done à eludier les projets exislants, en lenant compte de limportance fondamentale de la construction de ces lignes pour le développement économique de la régrion.

\section{URBANISME.}

En ec qui concerne les problèmes d'urbanisme et d'assainissement urbain, la Commission juggeait bon d'éviter que les localités du São Francisco continuent à se développer d'une façon désordonnée, entraînant ainsi de plus grandes difficultés techniques et économiques pour leur développement rationnel. Aussi montre-t-elle les avantages qui seraient acquis si clle prenait a sa charge l'élaboration des projets d'urbanisme et d'alignement de ces localités ainsi que leurs systomes d'alimenlation en eau polable et leurs réseaux d'égouts, projets qui devront être exécutés simultanément pour chacune d'entre elles.

Pour l'exécution de ces travaux, pendant la première période quinquennale, la Commission traça le plan suivant:

a) Elaboration des plans d'urbanisme ef exécution des travaux d'assainissement urbain des villes de Pirapora, Juazeiro, Petrolina, Proprià el Penedo, tous ces travaux clant entièrement financés par la Commission;

b) Réalisation d'une façon progressive de ces mêmes travaux dans les autres localités de la vallée du São Francisco, en accord avec les préfectures intéressées et moyennant un financement partiel de leur part.

Les plans d'urbanisme des cinq villes mentionnées plus haut sont maintenant définis et les travaux d'installation des systèmes d'alimentation en eau se trouvent dans leur phase finale; tout ceci en conformité avec l'accord passé entre la Commission et le « Ministério da Saúde » (Ministère de la Santé), ce dernier ayant confié les travaux au « Serviço Especial de Saúde Pública » moyennant des crédits fournis chaque année par la Commission.

Sur le budget en cours, la seconde partic de ce plan a été mise en chantier et, dans ce but, la Commission a conclu des accords avec quatrevingt-deux préfectures riveraines appartenant aux Etats compris dans le bassin du São Francisco.

\section{Travaux D'assainissement.}

En ce qui concerne les travaux destinés à l'assainissement rural et au drainage, étant donnée la valeur économique des plaines du bas São Francisco, qui se prêtent à la culture intensive des céréales el principalement du riz, la Commission décida que les travaux commenceraient, dans celte partie de la vallée, par le drainage et le contrôle des eaux des affluents du tronçon correspondant du fleuve.

Pour l'exécution de ce programme, la Commission choisit, dans l'Etat de Alagoas, les bassins affluents des rivieres Itiuba, Baocica ef Marituba, et, dans l'Etat de Sergipe, les bassins des rivicies Betume, Propria et Cotinguiba. L'aménagement de ces plaines doit permettre la bonification de 20.000 ha environ de terres de première qualité.

L'accomplissement de ces travaux, durant la píriode quinquennale actuelle, est à la charge du «Departamento Nacional de Obras de Saneamento " (Département National des Travaux d'Assainissement), d'après l'accord passé avec cette Commission en 1952. D'après cet accord, l'assistance et l'aide aux agriculleurs riverains sont d'ailleurs placées directement sous la responsabilité de la Commission.

\section{EDvCation ET FORMATION PROFESSIONNEILE.}

En ce qui concerne l'éducation et l'enseignement professionnel, la Commission dirigea son action vers l'installation de fermes-écoles, l'orga- 
Fis. 9.

Ressources minières.

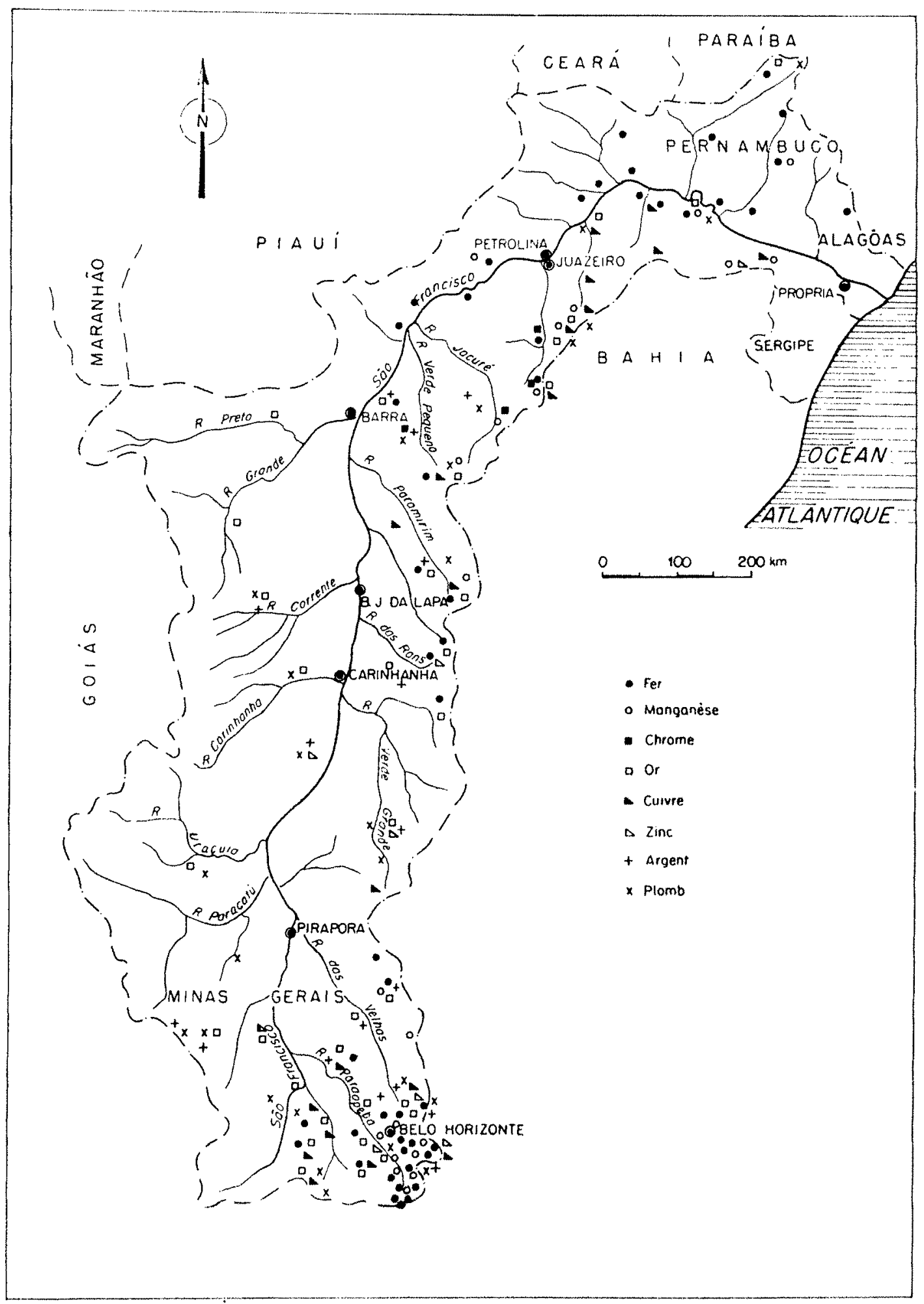

nisation de missions rurales ambulantes, et l'élablissement de cours d'apprentissage.

Pour la réalisation de ces travaux si importants, le budget en cours n'accordait à la Commission que les premiers crédits concernant l'aménagement d'une ferme-école dans le bas
São Francisco, à Pôrto Real de Colégio, dans l'Etal de Alagoas; sa construction dépend encore de la fin du proces pour la cession du terrain par le Ministère de l'Agriculture.

Dans les discussions qui ont eu lieu au Congrès National au sujet du projet du São Fran- 
cisco, on décida que les travalux prévus, dans le plan général d’aménagement du Sĩo Francisco, pour l'iducation et l'enseignement professionnel, ne seraient intensifiés qu'à partir de la seconde période quinquennale d'exćcution du plan, ce qui explique que des crédits plus substanticls n'aient pas encore été attribués à cet eflet.

En ce qui concerne les services de santé el d'assistance à la population régionale, la Commission décida :

(1) De créer des services généraux d'assistance au moyen d'unitis mobiles;

b) De construire et d'entretenir les unités qui composent le « Rède Hospitalar do São Francisco » (Réseau Hospitalier du São Francisco);

c) D'exiculer les travaux de prophylaxie contre lit malaria dans toutes les zones urbaines et rurales du bassin du São Francisco.

\section{Equipentent saxitaire.}

Les travaux généraux d'assistance que la Commission doit réaliser directement ont pour hut principal de mettre à la disposition des habitants de la région des services médicaux de toutes sortes, installés sur des navires, des véhicules terrestres on des avions, suivant le mode de transport adoplé : fluvial, terrestre ou aćrien. Cette action dynamique est justifiée par la dispersion des centres de population et par l'économie qu'elle comporte. La construction du premier navire prévu dans ce but, et destiné au moyen São Francisco, se trouve actuellement en adjudication.

Les travaux d'equipement el d'entretien du "Rède Hospitalar do São Francisco ", qui est composé de 62 unités sanitaires groupées en 11 districts, sont at la charge du « Servico Especial de Saúde Pública » (Service spécial de la Santé publique) d'après l'accord passé en 1950 entre la Commission et le «Ministério da Educaçio e Saúde (Ministère de l'Education et de la Sant(i), le financement de ces travaux incombant a la Commission, qui fournira annuellement les fonds nécessaires au S.E.S.P.

Lil poursuite des travaux de prophylaxie contre la malaria se trouve sous la responsabilité du \& Serviço National de Malaria », d'après le mème accord passí en 1950 entre la Commission el le "Ministério de Educacão e Saúde »; le financement des travaux revient à la Commission.

\section{Probleyes DE REBOISEMENT.}

En ce qui concerne les réserves forestières et le reboisement, la Commission, considérant la disparition progressive de la flore et de la faune de la vallée et exigeant des pouvoirs publics une solution appropriée, jugea nécessaire de prendre les mesures suivantes :

a) Créalion, à côté des colonies agricoles prévues dans les vallées occidentales du moyen Săo Francisco, de pares destinés at protéger la llore el la faune locales;

b) Execulion d'un vaste programme de conservation, de restauration et de création de forêts dans la région.

L'effet bienfaisant de la couverture végétale ainsi réalisée se fera sentir directement par l'augmentation des débits d'étiage, l'affaiblissement de l'érosion et la protection contre les avalanches.

\section{EQUHPMENT industriel at commercial.}

Enfin, en ce qui concerne le développement de la production régionale, la Commission, étant donné le plan progressif d'utilisation de ses ressources, imagina tout d'abord d'organiser des sections motorisées destinées à la mécanisation du travail, à la construction d'entrepôts, do silos et d'usines pour la préparation des denrées; d'établir un comptoir de revente des outils agricoles; d'organiser des postes de pêche; de construire des abattoirs industriels et d'installer des laboratoires pour la fabrication de vaccins anti-aphteux et l'exécution de travaux destinés à la protection sanitaire des animaux et des plantes.

Les exploitations agricoles de Januária, dans l'Etat de Minas Gerais, de Baixío de Irécê, dans l'Etat de Bahia, et une exploitation du bas São Francisco, sont maintenant aménagées en vue de la mécanisation du travail dans la région. Les premiers résultats obtenus sont des plus encourageants, notamment dans la culture du riz dans les plaines et les lagunes du São Francisco, où une véritable révolution est faite actuellement dans les méthodes routinières pratiquées jusqu'ici. Le programme d'aménagement de cette région comprend aussi l'installation d'usines achetées par la Commission et destinées, sous forme de coopératives, à la transformation de produits.

Cet exposé montre que la Commission de la vallée du São Francisco, qui atteindra sa sixième année à la fin du budget en cours, n'a pas ménagé ses efforts pour remplir la mission que lui a confiée la Constitution de la République; elle espère voir, à la fin du délai déterminé par la Carta Magna, la vallée du São Francisco prendre place parmi les régions les plus développées du pays. 